\title{
History-dependent operators and prox-regular sweeping processes
}

\author{
Florent Nacry ${ }^{1 *}$ (D) and Mircea Sofonea ${ }^{1}$
}

${ }^{*}$ Correspondence:

florent.nacry@univ-perp.fr

'Laboratoire de Modélisation

Pluridisciplinaire et Simulations,

Université Perpignan, 52 Avenue

Paul Alduy, Perpignan (66860),

France

\begin{abstract}
We consider an abstract inclusion in a real Hilbert space, governed by an almost history-dependent operator and a time-dependent multimapping with prox-regular values. We establish the unique solvability of the inclusion under appropriate assumptions on the data. The proof is based on the arguments of monotonicity, fixed point, and prox-regularity. We then use our result in order to deduce some direct consequences, including an existence and uniqueness result for a class of sweeping processes associated with prox-regular sets. Finally, we provide an example in a finite dimensional case inspired by a rheological model in solid mechanics.
\end{abstract}

MSC: 47J22; 34G25; 45G10; 74G20

Keywords: Time-dependent inclusion; Prox-regular set; Normal cone; Sweeping process; History-dependent operator; Existence and uniqueness; Rheological model

\section{Introduction}

A large variety of boundary valued problems arising in mechanics, physics, and engineering sciences lead, in a weak formulation, to nonlinear inclusions. Their solvability involves arguments coming from nonlinear, set-valued, convex, and nonsmooth analysis, among others. Currently, there is a growing interest in the solution of inclusions governed by a special class of operators, the so-called almost history-dependent operators. Such kind of problems arise in the study of different constitutive laws used in the viscoelasticity and viscoplasticity. They also describe the frictional or frictionless contact between a deformable body and an obstacle. References in the field include $[1,5,19,20]$. There, existence and uniqueness results have been provided by using a fixed point theorem for almost historydependent operators.

Nevertheless, the inclusions studied in the previously cited papers have been associated with a family of convex sets. Removing the convexity in the study of the corresponding inclusions leads to important mathematical difficulties and gives rise to new and challenging mathematical problems. This can be achieved through the class of prox-regular sets (also known as positively reached, weakly convex, $\mathcal{O}(2)$-convex, $\varphi$-convex, proximally smooth (see, e.g., [11] and the references therein)). Recall that a closed set is said to be prox-regular [23] provided that its metric projection is single-valued and continuous on a suitable enlargement of the set. Prox-regular sets share important properties with con-

(c) The Author(s) 2022. This article is licensed under a Creative Commons Attribution 4.0 International License, which permits use, sharing, adaptation, distribution and reproduction in any medium or format, as long as you give appropriate credit to the original author(s) and the source, provide a link to the Creative Commons licence, and indicate if changes were made. The images or other third party material in this article are included in the article's Creative Commons licence, unless indicated otherwise in a credit line to the material. If material is not included in the article's Creative Commons licence and your intended use is not permitted by statutory regulation or exceeds the permitted use, you will need to obtain permission directly from the copyright holder. To view a copy of this licence, visit http://creativecommons.org/licenses/by/4.0/. 
vex sets, including smoothness of distance function, hypomonotonicity of normals, and global Lipschitz property of metric projection (see Sect. 2). Prox-regularity property has been recognized as a key concept in variational analysis and its applications [16, 24, 28]. The good behavior of metric projection and distance function for prox-regular sets is extensively involved in the study of Moreau's sweeping processes (that is, differential inclusions driven by the normal cone of a moving set $[15,17,18])$ as shown in $[3,6-8,10,12-$ $14,21,27,29]$ and the references therein. However, at the best of our knowledge, there are few literature works concerning history-dependent inclusions governed by prox-regular sets.

The aim of this current paper is to fill this gap. Indeed, here we consider a version of the history-dependent inclusion considered in [19], associated with a family of prox-regular sets. We provide an existence and uniqueness result and, to this end, we use various properties of the prox-regular sets, including the properties of the projection operators and proximal normal cone. Extending the results in [19] to the nonconvex case represents the main trait of novelty in our work.

The structure of the inclusion we consider in this paper is motivated by the study of constitutive laws in solid mechanics, as we show in what follows. Let $d \in\{2,3\}$ and denote by $\mathbb{S}^{d}$ the space of second order symmetric tensors on $\mathbb{R}^{d}$ or, equivalently, the space of symmetric matrices of order $d$. Recall that the inner product and the Euclidean norm on $\mathbb{S}^{d}$ are defined by

$$
\boldsymbol{\sigma} \cdot \boldsymbol{\tau}=\sigma_{i j} \tau_{i j}, \quad\|\boldsymbol{\tau}\|=(\boldsymbol{\tau} \cdot \boldsymbol{\tau})^{\frac{1}{2}} \quad \forall \boldsymbol{\sigma}=\left(\sigma_{i j}\right), \boldsymbol{\tau}=\left(\tau_{i j}\right) \in \mathbb{S}^{d},
$$

where the indices $i, j$ run between 1 and $d$ and the summation convention over repeated indices is used. Consider a constitutive law derived by using the following rheological arguments.

1) The model is obtained by connecting in series an elastic model with a viscoelastic (or viscoplastic) model. Then, at each moment $t$ in the interval of interest $I$, the strain field satisfies the equality

$$
\boldsymbol{\varepsilon}(t)=\boldsymbol{\varepsilon}_{1}(t)+\boldsymbol{\varepsilon}_{2}(t),
$$

where $\varepsilon_{1}$ and $\varepsilon_{2}$ represent the strain field in the elastic and the viscoelastic (or viscoplastic) model, respectively. We refer to $\boldsymbol{\varepsilon}_{1}$ and $\boldsymbol{\varepsilon}_{2}$ as the "regular" and "irregular" strain.

2) The regular strain satisfies the equality

$$
\boldsymbol{\varepsilon}_{1}(t)=B \boldsymbol{\sigma}(t)
$$

where $\sigma=\sigma(t)$ denotes the stress field and $B: \mathbb{S}^{d} \rightarrow \mathbb{S}^{d}$ represents the compliance operator which could be nonlinear. This operator is supposed to be inversible, and its inverse will be denoted by $A$, i.e., $B^{-1}=A$.

3) On the other hand, we assume that the irregular strain field is such that

$$
\boldsymbol{\varepsilon}_{2}(t) \in N(C(t) ; \sigma(t)+\mathcal{R} \boldsymbol{\sigma}(t)),
$$


where $C(t)$ is a subset of $\mathbb{S}^{d}$ to be defined and $\mathcal{R}$ is a memory operator. A popular example of such an operator is given by

$$
\mathcal{R}(\sigma)=\int_{0}^{t} \mathcal{D}(t-s) \boldsymbol{\sigma}(s) d s
$$

where $\mathcal{D}(\cdot)$ denotes a given relaxation tensor. Moreover, for any $\omega \in \mathbb{S}^{d}$, notation $N(C(t), \omega)$ represents a set of $\mathbb{S}^{d}$ which depends on $C(t)$. Note that (3) shows that at each time moment $t$ the irregular strain depends on the current value of the stress (i.e., $\sigma(t))$ and the history of the stress process (described by the term $\mathcal{R} \sigma(t)$ ).

We now combine relations (1)-(3) to deduce that

$$
\boldsymbol{\varepsilon}(t) \in B \boldsymbol{\sigma}(t)+N(C(t) ; \boldsymbol{\sigma}(t)+\mathcal{R} \boldsymbol{\sigma}(t)) .
$$

A concrete example of constitutive law of the form (4) can be obtained by taking $\mathcal{R} \equiv 0$ and $N(C(t), \omega)=\partial \psi_{C(t)}(\omega)$ for any $\omega \in \mathbb{S}^{d}$, where $C(t) \subset \mathbb{S}^{d}$ is a given convex set (say the von Mises convex) and $\partial \psi_{C(t)}$ represents the Moreau-Rockafellar subdifferential of the indicator function (in the sense of convex analysis) $\psi_{C(t)}$ of the set $C(t)$. This leads to the well-known Hencky law

$$
\boldsymbol{\varepsilon}(t) \in B \sigma(t)+\partial \psi_{C(t)}(\sigma(t))
$$

see, e.g., $[22,26]$ and the references therein. Note that in (5) we assume that the convex $C$ is time-dependent, and this could arise when $C$ depends on the temperature field, for instance.

Now, we are looking for a stress function $\sigma$ which, applied to the deformable body, keeps it in equilibrium, i.e., the strain field vanishes. Then, at each moment $t \in I$, we have

$$
-B \sigma(t) \in N(C(t) ; \sigma(t)+\mathcal{R} \sigma(t))
$$

and, using the notation $\boldsymbol{\omega}=\boldsymbol{\varepsilon}_{1}$ combined with equalities (2) and $B^{-1}=A$, we find that

$$
-\omega(t) \in N(C(t) ; A \omega(t)+\mathcal{R} A \omega(t)) .
$$

Therefore, with the notation $\mathcal{R} A \omega(t)=\mathcal{S} \omega(t)$ we arrive at the following inclusion problem.

Problem 1 Find a regular strain function $\omega: I \rightarrow \mathbb{S}^{d}$ such that

$$
-\omega(t) \in N(C(t) ; A \omega(t)+\mathcal{S} \omega(t)) \quad \text { for all } t \in I .
$$

Motivated by the above mechanical problem, in this paper we shall study inclusions of the form (6) in the abstract framework of real Hilbert spaces, under the assumption that $C(t)$ represents a family of prox-regular sets and $\mathcal{S}$ is an almost history-dependent operator.

The rest of the manuscript is organized as follows. In Sect. 2 we recall some notation and preliminaries which are used in the rest of the paper. In Sect. 3 we introduce the abstract history-dependent inclusion and state the main existence and uniqueness result, 
Theorem 3.1, together with its consequences. The proof of the theorem is given in Sect. 4 based on a fixed point argument. Finally, in Sect. 5 we provide an example of Problem 1 for which our abstract results work.

\section{Notation and preliminaries}

In the whole paper, all vector spaces will be real vector spaces. We use $\mathbb{R}_{+}$for the set of nonnegative reals, that is, $\mathbb{R}_{+}:=[0,+\infty)$. The letter $T$ stands for an extended nonnegative real, i.e., $T \in \mathbb{R}_{+} \cup\{+\infty\}$ and $I:=[0, T] \cap \mathbb{R}_{+}$. In what follows $X$ is a (real) Hilbert space endowed with its inner product $(\cdot, \cdot)_{X}$ and its associated norm $\|\cdot\|_{X}$. The open (resp. closed) ball with respect to the norm $\|\cdot\|_{X}$ centered at $x \in X$ with radius $r>0$ is denoted by $B(x, r)$ (resp. $B[x, r])$. The letter $\mathbb{U}_{X}$ (resp. $\mathbb{B}_{X}$ ) stands for the open (resp. closed) unit ball of $X$ centered at the origin $0_{X}$, that is, $\mathbb{U}_{X}:=B\left(0_{X}, 1\right)$ (resp. $\left.\mathbb{B}_{X}:=B\left[0_{X}, 1\right]\right)$. The strong and weak convergences in $X$ will be denoted by $\rightarrow$ and $\rightarrow$, respectively, and are considered as $n \rightarrow \infty$, even if we do not mention it explicitly. Recall that $\|\cdot\|_{X}$ enjoys the so-called sequential Kadec-Klee property, that is, every sequence $\left(x_{n}\right)_{n} \subset X$ satisfying $x_{n} \rightarrow x$ along with $\left\|x_{n}\right\|_{X} \rightarrow\|x\|_{X}$ for some $x \in X$ converges strongly to $x$.

Projections and nonlinear operators The metric projection multimapping Proj $_{S}: X \rightrightarrows X$ associated with a nonempty subset $S \subset X$ is defined as

$$
\operatorname{Proj}_{S}(x):=\left\{y \in S: d_{S}(x)=\|x-y\|_{X}\right\} \quad \text { for all } x \in X
$$

where $d_{S}(\cdot)$ (or $\left.d(\cdot, S)\right)$ is the distance function from $S$, that is,

$$
d_{S}(x):=: d(x, S):=\inf _{y \in S}\|x-y\|_{X} \quad \text { for all } x \in X .
$$

When the $\operatorname{set} \operatorname{Proj}_{S}(\bar{x})$ is reduced to a singleton for some vector $\bar{x} \in X$, we say that the metric projection of $\bar{x}$ on $S$ is well defined. In such a case, the unique element of $\operatorname{Proj}_{S}(\bar{x})$ is denoted by $\operatorname{proj}_{S}(\bar{x})$ or $P_{S}(\bar{x})$. It is an exercise to check that, for any $x, x^{\prime} \in X$,

$$
x^{\prime} \in \operatorname{Proj}_{S}(x) \quad \Longleftrightarrow \quad x^{\prime} \in S \quad \text { and } \quad\left(x-x^{\prime}, y-x^{\prime}\right)_{X} \leq \frac{1}{2}\left\|y-x^{\prime}\right\|_{X}^{2} \quad \text { for all } y \in S .
$$

It is known (and not difficult to establish) that the multimapping $\operatorname{Proj}_{S}(\cdot)$ is monotone, that is,

$$
\left(p_{1}-p_{2}, x_{1}-x_{2}\right)_{X} \geq 0
$$

for every $x_{1}, x_{2} \in X, p_{1} \in \operatorname{Proj}_{S}\left(x_{1}\right)$, and $p_{2} \in \operatorname{Proj}_{S}\left(x_{2}\right)$.

In the development below, the concept of strong monotonicity of operators will be needed. Recall that an operator $A: X \rightarrow X$ is said to be strongly monotone with constant $m_{A}>0$ provided that

$$
(A u-A v, u-v)_{X} \geq m_{A}\|u-v\|_{X}^{2} \quad \text { for all } u, v \in X
$$

Operators enjoying the Lipschitz property will be also used. We say that the operator $A$ : $Y \rightarrow X$ is Lipschitz continuous with constant $L_{A}>0$ on $Y \subset X$ provided that

$$
\|A u-A v\|_{X} \leq L_{A}\|u-v\|_{X} \quad \text { for all } u, v \in Y .
$$


The following result on strongly monotone Lipschitz continuous operators will be crucial in our study.

Proposition 2.1 Let $A: X \rightarrow X$ be a strongly monotone Lipschitz continuous operator with respective constants $m_{A}>0$ and $L_{A}>0$. Then $A: X \rightarrow X$ is invertible, and its inverse $A^{-1}$ : $X \rightarrow X$ is also strongly monotone and Lipschitz continuous with respective $\frac{m_{A}}{L_{A}^{2}}$ and $\frac{1}{m_{A}}$, respectively.

For the proof of Proposition 2.1, we refer to [25, Theorem 1.24].

Proximal normal cone We now assume that $S \subset X$ and $U$ is a nonempty open subset of $X$. Then the proximal normal cone to $S$ at $x \in X$ is defined as the set

$$
N(S ; x):= \begin{cases}\left\{v \in X: \exists r>0, x \in \operatorname{Proj}_{S}(x+r v)\right\} & \text { if } x \in S, \\ \emptyset & \text { otherwise. }\end{cases}
$$

For each $x \in S$, it is known that $N(S ; x)$ is a convex cone (not necessarily closed), containing $0_{X}$. Further, it can be checked that, for given $(v, x) \in X \times S$, the inclusion $v \in N(S ; x)$ holds if and only if there is some real $\sigma \geq 0$ such that

$$
\left(v, x^{\prime}-x\right)_{X} \leq \sigma\left\|x^{\prime}-x\right\|_{X}^{2} \quad \text { for all } x^{\prime} \in S
$$

From the above definition, it is not difficult to see that that for any $v \in X$ with $\operatorname{Proj}_{S}(v) \neq \emptyset$ the following inclusion holds:

$$
v-w \in N(S ; w) \quad \text { for all } w \in \operatorname{Proj}_{S}(v) .
$$

We conclude this part devoted to the proximal normal cone by recalling that if $S$ is convex, then the following equality holds:

$$
N(S ; x)=\left\{v \in X:\left(v, x^{\prime}-x\right)_{X} \leq 0, \forall x^{\prime} \in S\right\} \quad \text { for all } x \in S .
$$

Prox-regular sets We now recall the notion of prox-regular sets in Hilbert spaces. For historical comments, proofs, and further results, we refer to the survey by G. Colombo and L. Thibault [11] (see also the forthcoming monograph [28]) and the references therein.

Definition 2.2 Let $S$ be a nonempty closed subset of $X$, and let $r \in(0,+\infty]$. One says that $S$ is $r$-prox-regular (or uniformly prox-regular with constant $r$ ) whenever for all $x \in S$, $v \in N(S ; x) \cap \mathbb{B}_{X}$ and $t \in(0, r]$ one has

$$
x \in \operatorname{Proj}_{S}(x+t v) .
$$

Concerning this definition we have the following comments. First, note that if $S$ is $r$ prox-regular, then it is $r^{\prime}$-prox-regular for any $0<r^{\prime}<r$. Further, it is known that the class of $\infty$-prox-regular subsets of $X$ is nothing but the class of nonempty closed convex sets of $X$. 
Given a nonempty subset $S \subset X$, we denote by $U_{r}(S)$ and $\operatorname{Enl}_{r}(S)$ the $r$-open and closed enlargement of $S$, that is,

$$
U_{r}(S):=\left\{x \in X: d_{S}(x)<r\right\}
$$

and

$$
\operatorname{Enl}_{r}(S):=\left\{x \in X: d_{S}(x) \leq r\right\}
$$

Moreover, if $r:=+\infty$, we set $1 / r:=0$ and $U_{r}(S):=X$.

The following theorem provides some useful characterizations and properties of uniform prox-regular sets.

Theorem 2.3 Let $S$ be a nonempty closed subset of $X$. The following assertions are equivalent for any extended real $r \in(0,+\infty]$.

(a) The set $S$ is r-prox-regular.

(b) For all $x_{1}, x_{2} \in S$ and $\zeta \in N\left(S ; x_{1}\right) \cap \mathbb{B}_{X}$, one has

$$
\left(\zeta, x_{2}-x_{1}\right)_{X} \leq \frac{1}{2 r}\left\|x_{1}-x_{2}\right\|_{X}^{2}
$$

(c) For all $x_{1}, x_{2} \in S, \zeta_{1} \in N\left(S ; x_{1}\right) \cap \mathbb{B}_{X}$, and $\zeta_{2} \in N\left(S ; x_{2}\right) \cap \mathbb{B}_{X}$, one has

$$
\left(\zeta_{1}-\zeta_{2}, x_{1}-x_{2}\right)_{X} \geq-\frac{1}{r}\left\|x_{1}-x_{2}\right\|_{X}^{2}
$$

(d) For any $0<s<r, \operatorname{proj}_{S}(x)$ is well defined for every $x \in U_{S}(S)$ and the mapping $\operatorname{proj}_{S}(\cdot)$ is $(1-s / r)^{-1}$-Lipschitz continuous therein, i.e.,

$$
\left\|\operatorname{proj}_{S}(x)-\operatorname{proj}_{S}\left(x^{\prime}\right)\right\|_{X} \leq \frac{1}{1-s / r}\left\|x-x^{\prime}\right\|_{X} \quad \text { for all } x, x^{\prime} \in U_{S}(S) .
$$

(e) The function $x \mapsto d_{S}^{2}(x)$ is $C^{1,1}$ on $U_{r}(S)$ and

$$
\nabla d_{S}^{2}(x)=2\left(x-\operatorname{proj}_{S}(x)\right) \quad \text { for all } x \in U_{r}(S)
$$

(f) For any $0<s<r$, the function $x \mapsto d_{S}^{2}(x)+\frac{s}{r-s}\|x\|_{X}^{2}$ is convex on any open convex subset of $U_{s}(S)$.

Let $\mathcal{N}$ be any of the normal cones in the sense of the Fréchet, Mordukhovich, or Clarke (see, e.g., $[9,16,28]$ for the definitions and basic properties). It is known that assertions $(b)$ and $(c)$ with the truncated normal cone $\mathcal{N}(S ; \cdot) \cap \mathbb{B}_{X}$ in place of the truncated proximal one $N(S ; \cdot) \cap \mathbb{B}_{X}$ are also equivalent to the $r$-prox-regularity of $S$. Further, any $r$-prox-regular set $S$ enjoys the following normal regularity:

$$
\mathcal{N}(S ; x)=N(S ; x) \quad \text { for all } x \in S \text {. }
$$

Moreover, taking $r=\infty$ in Theorem 2.3 leads to the following result. 
Corollary 2.4 Let $S$ be a nonempty closed subset of $X$. The following assertions are equivalent.

(a) The set $S$ is convex.

(b) For all $x_{1}, x_{2} \in S, \zeta_{1} \in N\left(S ; x_{1}\right)$, and $\zeta_{2} \in N\left(S ; x_{2}\right)$, one has

$$
\left(\zeta_{1}-\zeta_{2}, x_{1}-x_{2}\right)_{X} \geq 0
$$

(c) For every $x \in X, \operatorname{proj}_{S}(x)$ is well defined and the mapping $\operatorname{proj}_{S}(\cdot)$ is 1-Lipschitz continuous, i.e.,

$$
\left\|\operatorname{proj}_{S}(x)-\operatorname{proj}_{S}\left(x^{\prime}\right)\right\|_{X} \leq\left\|x-x^{\prime}\right\|_{X}
$$

(d) The function $x \mapsto d_{S}^{2}(x)$ is $C^{1,1}$ on $X$ and

$$
\nabla d_{S}^{2}(x)=2\left(x-\operatorname{proj}_{S}(x)\right) \quad \text { for all } x \in X .
$$

(e) The function $x \mapsto d_{S}^{2}(x)$ is convex on $X$.

We now proceed with two results strongly involved in the proof of our main theorem below. The first one is related to inclusion (10) for prox-regular sets.

Lemma 2.5 ([3]) Let $r \in(0,+\infty]$, S be an $r$-prox-regular set of $X$, and let $x, x^{\prime} \in X$.If $x-x^{\prime} \in$ $N\left(S ; x^{\prime}\right)$ and $\left\|x-x^{\prime}\right\|_{X} \leq r\left(\right.$ resp. $\left.\left\|x-x^{\prime}\right\|_{X}<r\right)$, then $x^{\prime} \in \operatorname{Proj}_{S}(x)\left(\right.$ resp. $\left.x^{\prime}=\operatorname{proj}_{S}(x)\right)$.

The second result deals with some convergence properties of prox-regular sets.

Lemma 2.6 Let $\left(S_{n}\right)_{n \in \mathbb{N}}$ be a sequence of $r$-prox-regular subsets of $X$ for some $r \in(0,+\infty]$, and let also $S$ be an r-prox-regular subset of $X$. Then, for every $x \in U_{r}(S)$ such that $d\left(x, S_{n}\right) \rightarrow d(x, S)$, one has that $\operatorname{proj}_{S_{n}}(x)$ is well defined for $n \in \mathbb{N}$ large enough and

$$
\operatorname{proj}_{S_{n}}(x) \rightarrow \operatorname{proj}_{S}(x) \quad \text { in } X .
$$

Proof Fix any $x \in U_{r}(S)$ and assume that $d\left(x, S_{n}\right) \rightarrow d(x, S)$. Take real $0<s^{\prime}<r$ such that $d(x, S)<s^{\prime}$ and take $s \in \mathbb{R}$ such that $s^{\prime}<s<r$. Since $d(x, S)<s^{\prime}$, there is an integer $N \in \mathbb{N}$ such that for every integer $n \geq N$ one has $d\left(x, S_{n}\right)<s^{\prime}$. Set $V:=B\left(x, s-s^{\prime}\right)$ and fix any integer $n \geq N$. For every $y \in V$, we have

$$
d\left(y, S_{n}\right) \leq d\left(x, S_{n}\right)+\|y-x\|_{X}<s^{\prime}+s-s^{\prime}=s,
$$

so $V \subset U_{s}\left(S_{n}\right)$ and, similarly, $V \subset U_{s}(S)$. Define the function $f_{n}: V \rightarrow \mathbb{R}$ by

$$
f_{n}(y):=d\left(y, S_{n}\right)^{2}+\frac{s}{r-s}\|y\|_{X}^{2} \quad \text { for all } y \in V
$$

and the function $f: V \rightarrow \mathbb{R}$ by

$$
f(y):=d(y, S)^{2}+\frac{s}{r-s}\|y\|_{X}^{2} \quad \text { for all } y \in V .
$$


According to Theorem 2.3, the functions $f_{n}$ and $f$ are convex and Fréchet differentiable on $V$. Moreover,

$$
\nabla f_{n}(y)=2\left(y-\operatorname{proj}_{S_{n}}(y)\right)+\frac{2 s}{r-s} y, \quad \nabla f(y)=2\left(y-\operatorname{proj}_{S}(y)\right)+\frac{2 s}{r-s} y
$$

for each $y \in V$. Set $v_{n}:=x-\operatorname{proj}_{S_{n}}(x)$ for every $n \geq N$. Note that the sequence $\left(v_{n}\right)_{n \geq N}$ is bounded since, by assumption, $\left(d_{S_{n}}(x)\right)_{n \geq N}$ converges in $\mathbb{R}$. Let $\left(v_{s(n)}\right)_{n \geq N}$ be any weakly convergent subsequence of the sequence $\left(v_{n}\right)_{n \geq N}$. Let $v$ be its limit with respect to the weak topology on $X$. Fix any $z \in V$. Keeping in mind that $f_{s(n)}(\cdot)$ is a convex function, for each integer $n \geq N$, we may write

$$
\left(\nabla f_{s(n)}(x), z-x\right)_{X} \leq f_{s(n)}(z)-f_{s(n)}(x)
$$

Then, passing to the limit as $n \rightarrow \infty$, we find that

$$
\left(2 v+\frac{2 s}{r-s} x, z-x\right)_{X} \leq f(z)-f(x)
$$

Since $z \in V$ is arbitrary, we get $2 v+\frac{2 s}{r-s} x \in \partial f(x)=\{\nabla f(x)\}$, i.e.,

$$
\nabla f(x)=2 v+\frac{2 s}{r-s} x
$$

Coming back to (11), we see that $v=x-\operatorname{proj}_{S}(x)$. Therefore, the whole sequence $\left(v_{n}\right)_{n \geq N}$ converges weakly in $X$ to $x-\operatorname{proj}_{S}(x)$. On the other hand, we obviously have

$$
\left\|v_{n}\right\|_{X}=d\left(x, S_{n}\right) \rightarrow d(x, S)=\left\|x-\operatorname{proj}_{S}(x)\right\|_{X}
$$

These two ingredients allow us to apply the Kadec-Klee property of the norm of $X$ to obtain the strong convergence $v_{n} \rightarrow x-\operatorname{proj}_{S}(x)$ in $X$. It results from the above that the whole sequence $\left(v_{n}\right)_{n \geq N}$ converges to $x-\operatorname{proj}_{S}(x)$ in $X$, which means that $\operatorname{proj}_{S_{n}}(x) \rightarrow$ $\operatorname{proj}_{S}(x)$ in $X$. The proof is then complete.

Examples and counter-examples Theorem 2.3 shows that prox-regular and convex sets share many properties, including the differentiability of distance function, the existence of nearest points, and (hypo)monotonicity of normals, among others. This naturally led several authors to study preservation of prox-regularity under various set operations. In what follows we shall use an example based on the following general result.

Lemma 2.7 ([28]) If $S_{1}$ and $S_{2}$ are $r$-prox-regular sets of $X$ for some $r>0$, then $S_{1} \cup S_{2}$ is $\min \{g / 2, r\}$-prox-regular whenever $g:=\inf _{\left(c_{1}, c_{2}\right) \in S_{1} \times S_{2}}\left\|c_{1}-c_{2}\right\|>0$.

Remark 2.8 For the convenience of the reader, we also provide the following counterexamples.

a) [11] Given real $r>0$, there is an $r$-prox-regular set $S$ of $\mathbb{R}^{2}$ such that $Q:=S \cap \mathbb{R} \times\{0\}$ fails to be uniformly (even locally !) prox-regular. 
b) The inverse image of a uniformly prox-regular set by a continuous linear mapping may fail to be prox-regular. Indeed, the above sets $Q$ and $S$ satisfy $A^{-1}(Q)=S$ with $A: \mathbb{R} \rightarrow \mathbb{R}^{2}$ defined by

$$
A(x):=(x, 0) \quad \text { for all } x \in \mathbb{R}
$$

c) [4, Example 7] The direct image of a uniformly prox-regular set by a continuous linear mapping may fail to be prox-regular.

d) [2] The sublevel $\{f \leq 0\}$ (resp. the level $\{f=0\}$ ) is not prox-regular even for smooth functions. This can be seen in a straightforward way with the function $f: \mathbb{R}^{2} \rightarrow \mathbb{R}$ defined by

$$
f(x, y):=x y \quad \text { for all }(x, y) \in \mathbb{R}^{2} .
$$

Finally, we recall that, despite the above counter-examples, sufficient conditions ensuring the prox-regularity of $C:=\left\{f_{1} \leq 0, \ldots, f_{p} \leq 0, h_{1}=0, \ldots, h_{q}=0\right\}$ are developed in [2] in the framework of Hilbert spaces.

History-dependent and almost history-dependent operators For a normed space $(Y, \|$. $\|_{Y}$ ), we denote by $C(I ; Y)$ the space of continuous functions defined on $I$ with values in $Y$, i.e.,

$$
C(I ; Y)=\{v: I \rightarrow Y: v \text { is continuous }\}
$$

The case $T>0$ (i.e., $I=[0, T])$ leads to the space $C([0, T]$; $Y$ ) which is a normed space equipped with the norm $\|\cdot\|_{C([0, T] ; Y)}$ defined by

$$
\|v\|_{C([0, T] ; Y)}:=\max _{t \in[0, T]}\|v(t)\|_{Y} \quad \text { for all } v \in C([0, T] ; Y) .
$$

It is well known that $C([0, T] ; Y)$ is a Banach space whenever $Y$ is a Banach space. The case $I=\mathbb{R}_{+}$leads to the space $C\left(\mathbb{R}_{+} ; Y\right)$. If $Y$ is a Banach space, then $C\left(\mathbb{R}_{+} ; Y\right)$ can be organized in a canonical way as a Fréchet space, i.e., a complete metric space in which the corresponding topology is induced by a countable family of seminorms.

The vector space of continuously differentiable functions on $I$ with values in $Y$ is denoted by $C^{1}(I ; Y)$. Obviously, for any function $v: I \rightarrow Y$, the inclusion $v \in C^{1}(I ; Y)$ holds if and only if $v \in C(I ; Y)$ and $\dot{v} \in C(I ; Y)$. Here and in what follows, $\dot{v}(\cdot)$ stands for the derivative of the function $v(\cdot)$. For a function $v \in C^{1}(I ; Y)$, the following equality will be used in the next section of this manuscript:

$$
v(t)=\int_{0}^{t} \dot{v}(s) d s+v(0) \quad \text { for all } t \in I .
$$

Everywhere below, given two normed spaces $Y$ and $Z$ and an operator $\mathcal{S}: C(I ; Y) \rightarrow$ $C(I ; Z)$, for any function $u \in C(I ; X)$, we use the shorthand notation $\mathcal{S} u(t)$ to represent the value of the function $\mathcal{S} u$ at the point $t \in I$, that is, $\mathcal{S} u(t):=(\mathcal{S} u)(t)$.

We end this section with two important classes of operators defined on the space of continuous functions. 
Definition 2.9 Let $\left(Y,\|\cdot\|_{Y}\right)$ (resp. $\left.\left(Z,\|\cdot\|_{Z}\right)\right)$ be a normed space, and let $K$ (resp. $\left.K^{\prime}\right)$ be a nonempty closed subset of $Y$ (resp. $Z$ ). An operator $\mathcal{S}: C(I ; K) \rightarrow C\left(I ; K^{\prime}\right)$ is called:

a) history-dependent (h.d. for short), if for any nonempty compact set $\mathcal{J} \subset I$, there exists $L_{\mathcal{J}}^{\mathcal{S}}>0$ such that, for all $u_{1}, u_{2} \in C(I ; K)$ and all $t \in \mathcal{J}$,

$$
\left\|\mathcal{S} u_{1}(t)-\mathcal{S} u_{2}(t)\right\|_{Z} \leq L_{\mathcal{J}}^{\mathcal{S}} \int_{0}^{t}\left\|u_{1}(s)-u_{2}(s)\right\|_{Y} d s
$$

b) almost history-dependent (a.h.d. for short), if for any nonempty compact set $\mathcal{J} \subset I$, there exist $l_{\mathcal{J}}^{\mathcal{S}} \in[0,1)$ and $L_{\mathcal{J}}^{\mathcal{S}}>0$ such that, for all $u_{1}, u_{2} \in C(I ; K)$ and all $t \in \mathcal{J}$,

$$
\left\|\mathcal{S} u_{1}(t)-\mathcal{S} u_{2}(t)\right\|_{Z} \leq l_{\mathcal{J}}^{\mathcal{S}}\left\|u_{1}(t)-u_{2}(t)\right\|_{Y}+L_{\mathcal{J}}^{\mathcal{S}} \int_{0}^{t}\left\|u_{1}(s)-u_{2}(s)\right\|_{Y} d s
$$

The next fixed point result makes clear the interest of such operators.

Theorem 2.10 Let $K$ be a nonempty closed subset of a Banach space $Y$, and let $\Lambda$ : $C(I ; K) \rightarrow C(I ; K)$ be an almost history-dependent operator. Then $\Lambda$ has a unique fixed point, i.e., there exists a unique element $\eta^{*} \in C(I ; K)$ such that $\Lambda \eta^{*}=\eta^{*}$.

A proof of Theorem 2.10 can be found in [26, pp. 41-45]. There, the main properties of history-dependent and almost history-dependent operators are presented together with various examples and applications.

\section{Problem statement and main results}

In this section we state an existence and uniqueness result for a time-dependent inclusion involving nonlinear operators. To this end we consider a nonempty closed bounded subset $K \subset X$, a multimapping $C: I \rightrightarrows X$, and two operators $A: X \rightarrow X$ and $\mathcal{S}: C(I ; X) \rightarrow C(I ; K)$. As usual, $\operatorname{Im}(C)$ denotes the range of $C(\cdot)$, that is,

$$
\operatorname{Im}(C):=\bigcup_{t \in I} C(t)
$$

With the above data and notation at hand, we introduce the following inclusion problem.

Problem 2 Find a continuous function $u: I \rightarrow X$ such that

$$
-u(t) \in N(C(t) ; A u(t)+\mathcal{S} u(t)) \quad \text { for all } t \in I .
$$

In the study of (14) we consider the following assumptions.

$(\mathcal{C})$ The multimapping $C: I \rightrightarrows X$ has $r$-prox-regular values for some real $r \in(0,+\infty]$ and, for every $t \in I$ and every sequence $\left(t_{n}\right)_{n \geq 1}$ of $I$ converging to $t$, one has

$$
d\left(u, C\left(t_{n}\right)\right) \rightarrow d(u, C(t)) \quad \text { for all } u \in U_{r}(C(t))
$$

(A) The mapping $A$ is $m_{A}$-strongly monotone and $L_{A}$-Lipschitz continuous for some reals $m_{A}, L_{A}>0$. 
$(\mathcal{S})$ For any nonempty compact set $\mathcal{J} \subset I$, there exist $l_{\mathcal{J}}^{\mathcal{S}}>0$ and $L_{\mathcal{J}}^{\mathcal{S}}>0$ such that, for all $u_{1}, u_{2} \in C(I ; X)$ and $t \in \mathcal{J}$, inequality (13) holds.

Note that, using Lemma 2.6, it follows that the Wijsman-type convergence (15) is equivalent to the convergence in $X$

$$
\operatorname{proj}_{S_{n}}(x) \rightarrow \operatorname{proj}_{S}(x) \quad \text { for all } x \in U_{r}(S)
$$

Our main result in the study of Problem 2 that we state here and prove in the next section is the following.

Theorem 3.1 Assume $(\mathcal{C}),(\mathcal{A})$, and $(\mathcal{S})$. Assume also that

$$
\begin{aligned}
& m_{A}<\min \left\{L_{A}, L_{A}^{2 / 3}\right\}, \\
& s:=\sup _{x \in \operatorname{Im}(C)-K}\left\|A^{-1}(x)\right\|_{X}<\frac{m_{A}^{3}}{L_{A}^{2}}\left(1-\sqrt{1-\frac{m_{A}^{4}}{L_{A}^{4}}}\right) r
\end{aligned}
$$

along with

$$
\frac{m_{A}}{2}>\frac{2 s L_{A}^{2}}{r}
$$

In addition, assume that for any nonempty compact set $\mathcal{J} \subset I$ the following smallness condition holds:

$$
\left(\frac{1}{2 m_{A}}+\frac{2 s}{r}\right)^{1 / 2} l_{\mathcal{J}}^{\mathcal{S}}<\left(\frac{m_{A}}{2}-\frac{2 s L_{A}^{2}}{r}\right)^{1 / 2} .
$$

Then Problem 2 has at least a solution $u(\cdot)$. Moreover, the solution takes values in $s \mathbb{B}_{X}:=$ $\left\{s b: b \in \mathbb{B}_{X}\right\}$ and is the unique solution of Problem 2 with this property.

Remark 3.2 As mentioned in the introduction, Problem 2 has been already studied in [19] under the assumption that $C(t)$ is a nonempty closed convex moving set (that is, $\infty$-proxregular). It should be noted that if $r=\infty$, then estimate (18) obviously holds, (17) means that the operator $A^{-1}$ is bounded on the set $\operatorname{Im}(C)-K$, and (19) becomes

$$
l_{\mathcal{J}}^{\mathcal{S}}<m_{A}
$$

This inequality plays a crucial role for the well-posedness of Problem 2 driven by a convex set $C(t)$ studied in [19]. There, the set $K$ is possibly unbounded (say $K=X$ ), and there is no need to assume the boundedness of the operator $A^{-1}$.

In the rest of this section we present some consequences of Theorem 3.1.

Corollary 3.3 Assume $(\mathcal{C}),(\mathcal{A}),(16),(17)$, and (18). Assume also that $\mathcal{S}: C(I ; X) \rightarrow C(I ; K)$ is a history-dependent operator. Then Problem 2 has at least a solution $u(\cdot)$. Moreover, the solution takes values in $s \mathbb{B}_{X}:=\left\{s b: b \in \mathbb{B}_{X}\right\}$ and is the unique solution of Problem 2 with this property. 
Proof Since $\mathcal{S}$ is a history-dependent operator, Definition 2.9(a) guarantees that condition $(\mathcal{S})$ holds with $l_{\mathcal{J}}^{\mathcal{S}}=0$ for any compact $\mathcal{J} \subset I$. We deduce from here that in this case the smallness condition (19) is satisfied. Therefore, Corollary 3.3 is a direct consequence of Theorem 3.1.

Theorem 3.1 allows us to obtain an existence and uniqueness result for a first order sweeping process. To present it, besides the data $C, A$, and $\mathcal{S}$ and their associated assumptions $(\mathcal{C}),(\mathcal{A})$, and $(\mathcal{S})$, respectively, we consider an operator $B: X \rightarrow K$ and an element $u_{0}$ such that:

(B) $B: X \rightarrow X$ is a Lipschitz continuous operator with values in $Y \subset X$.

(K) $Y+K \subset K$.

(U) $u_{0} \in X$.

We are now in a position to introduce the following sweeping process.

Problem 3 Find a continuously differentiable function $u: I \rightarrow X$ such that

$$
\left\{\begin{array}{l}
-\dot{u}(t) \in N(C(t) ; A \dot{u}(t)+B u(t)+\mathcal{S} \dot{u}(t)) \quad \text { for all } t \in I, \\
u(0)=u_{0} .
\end{array}\right.
$$

Our first result in this section is the following.

Corollary 3.4 Assume that $(\mathcal{C}),(\mathcal{A}),(\mathcal{S}),(\mathcal{B}),(\mathcal{K}),(\mathcal{U}),(16),(17),(18)$, and (19). Then Problem 2 has at least a solution $u(\cdot)$. Moreover, its derivative $\dot{u}(\cdot)$ takes values in $s \mathbb{B}_{X}:=$ $\left\{s b: b \in \mathbb{B}_{X}\right\}$ and $u(\cdot)$ is the unique solution of Problem 3 with this property.

Proof We use assumption $(\mathcal{K})$ to introduce the operator $\widetilde{\mathcal{S}}: C(I ; X) \rightarrow C(I ; K)$ defined by

$$
\widetilde{\mathcal{S}} v(t):=B\left(\int_{0}^{t} v(s) d s+u_{0}\right)+\mathcal{S} v(t) \quad \text { for all } t \in I \text {, all } v \in C(I ; X) .
$$

Next, we consider the auxiliary problem of finding a function $v: I \rightarrow X$ such that

$$
-v(t) \in N(C(t) ; A v(t)+\widetilde{\mathcal{S}} v(t)) \quad \text { for all } t \in I .
$$

Let $L_{B}>0$ be a Lipschitz constant of the operator $B$. We use assumptions $(\mathcal{S})$ and $(\mathcal{B})$ to see that, for any nonempty compact set $\mathcal{J} \subset I$, any functions $v_{1}, v_{2} \in C(I ; X)$, and any $t \in I$, the following inequality holds:

$$
\left\|\widetilde{\mathcal{S}} v_{1}(t)-\widetilde{\mathcal{S}} v_{2}(t)\right\|_{X} \leq l_{\mathcal{J}}^{\mathcal{S}}\left\|v_{1}(t)-v_{2}(t)\right\|_{X}+\left(L_{B}+L_{\mathcal{J}}^{\mathcal{S}}\right) \int_{0}^{t}\left\|v_{1}(s)-v_{2}(s)\right\|_{X} d s
$$

Therefore, we are in a position to apply Theorem 3.1 in order to obtain the existence of a unique function $v \in C(I ; X)$ with values in $s \mathbb{B}_{X}$, which satisfies the time-dependent inclusion (21). Denote by $u: I \rightarrow X$ the function defined by

$$
u(t):=u_{0}+\int_{0}^{t} v(s) d s \quad \text { for all } t \in I
$$


Then (20)-(22) imply that $u$ is a solution of Problem 3 with regularity $u \in C^{1}(I ; X)$. This proves the existence part of the theorem. The uniqueness part follows from the unique solvability of auxiliary problem (21), guaranteed by Theorem 3.1.

A direct consequence of Corollary 3.4 is the following.

Corollary 3.5 Assume $(\mathcal{K}),(\mathcal{A}),(\mathcal{B}),(\mathcal{U}),(16),(17)$, and (18). Assume also that $\mathcal{S}$ : $C(I ; X) \rightarrow C(I ; K)$ is a history-dependent operator. Then Problem 2 has at least a solution $u(\cdot)$. Moreover, its derivative $\dot{u}(\cdot)$ takes values in $s \mathbb{B}_{X}:=\left\{s b: b \in \mathbb{B}_{X}\right\}$ and $u(\cdot)$ is the unique solution of Problem 2 with this property.

The proof of Corollary 3.5 follows from arguments similar to those used in the proof of Corollary 3.3 and, therefore, we skip it.

\section{Proof of Theorem 3.1}

The proof of Theorem 3.1 will be carried out in several steps that we present below. We start with a fixed point result for the projection mapping on a prox-regular set.

Lemma 4.1 Let $C$ be an $r$-prox-regular subset of $X$ for some $r \in(0,+\infty)$, and let $B: X \rightarrow X$ be an $m_{B}$-strongly monotone and $L_{B}$-Lipschitz continuous operator with $m_{B}<\min \left\{L_{B}, L_{B}^{2}\right\}$. Assume that

$$
\alpha:=\sup _{x \in C-K}\|B(x)\|_{X}<\rho\left(1-\sqrt{1-\rho m_{B}}\right) r=: \beta \quad \text { with } \rho:=\frac{m_{B}}{L_{B}^{2}}
$$

Then the following statements hold for any $\eta \in K$.

(a) For all $z \in C$ and all $\theta \in(0,1]$, one has $z-\theta B(z-\eta) \in \operatorname{Enl}_{\theta \alpha}(C) \subset U_{r}(C)$.

(b) For every $s \in[\rho \alpha, \beta]$, the mapping $C \ni z \mapsto \operatorname{proj}_{C}(z-\rho B(z-\eta))$ is a contraction on $C$ of constant $(1-s / r)^{-1}\left(1-m_{B} \rho\right)^{1 / 2}$.

(c) There exists a unique element $z_{\eta} \in C$ such that

$$
z_{\eta}=\operatorname{proj}_{C}\left(z_{\eta}-\rho B\left(z_{\eta}-\eta\right)\right)=\operatorname{proj}_{C}\left(z_{\eta}-B\left(z_{\eta}-\eta\right)\right) .
$$

Proof (a) Fix $\eta \in K$. Note that inclusion ( $a$ ) follows directly from the estimates

$$
d_{C}(z-\theta B(z-\eta)) \leq d_{C}(z)+\theta\|B(z-\eta)\|_{X} \leq \theta \sup _{x \in C-K}\|B(x)\|_{X}=\theta \alpha<r,
$$

valid for every $z \in C$ and every $\theta \in(0,1]$. This allows us to consider the mapping $\Lambda: C \rightarrow C$ defined by

$$
\Lambda z:=\operatorname{proj}_{C}(z-\rho B(z-\eta)) \text { for all } z \in C,
$$

where $\delta:=m_{B} \rho=\frac{m_{B}^{2}}{L_{B}^{2}} \in(0,1)$.

(b) We claim that $\Lambda$ is a contraction on $C$. First, note that

$$
1+\rho^{2} L_{B}^{2}-2 \rho m_{B}=1-\delta>0 .
$$


Pick any $s \in(\rho \alpha, \beta]$. We have $z-\rho B(z-\eta) \in \operatorname{Enl}_{\rho \alpha}(C) \subset U_{s}(C)$ for all $z \in C$. Fix any $z_{1}, z_{2} \in$ $C$ and set $u_{i}:=z_{i}-\eta$ for each $i \in\{1,2\}$. Using the definition of $\Lambda$ in (25) combined with the $\kappa:=(1-s / r)^{-1}$-Lipschitz property of $\operatorname{proj}_{C}(\cdot)$ on the $s$-open enlargement $U_{s}(C)$ of $C$, the $m_{B}$-strong monotonicity of $B$, and (26), we see that

$$
\begin{aligned}
\left\|\Lambda z_{1}-\Lambda z_{2}\right\|_{X}^{2} & \leq \kappa^{2}\left\|\left(z_{1}-\rho B\left(z_{1}-\eta\right)\right)-\left(z_{2}-\rho B\left(z_{2}-\eta\right)\right)\right\|_{X}^{2} \\
& =\kappa^{2}\left\|\left(u_{1}-u_{2}\right)-\rho\left(B u_{1}-B u_{2}\right)\right\|_{X}^{2} \\
& =\kappa^{2}\left\|u_{1}-u_{2}\right\|_{X}^{2}-2 \rho \kappa^{2}\left(u_{1}-u_{2}, B u_{1}-B u_{2}\right)_{X}+(\rho \kappa)^{2}\left\|B u_{1}-B u_{2}\right\|_{X}^{2} \\
& \leq \kappa^{2}\left(1+\rho^{2} L_{B}^{2}-2 \rho m_{B}\right)\left\|u_{1}-u_{2}\right\|_{X}^{2} \\
& =\kappa^{2}(1-\delta)\left\|u_{1}-u_{2}\right\|_{X}^{2}=\kappa^{2}(1-\delta)\left\|z_{1}-z_{2}\right\|_{X}^{2} .
\end{aligned}
$$

On the other hand, an elementary computation shows that

$$
\begin{aligned}
\kappa^{2}(1-\delta)<1 & \Longleftrightarrow 1-\frac{m_{B}^{2}}{L_{B}^{2}}<\left(1-\frac{s}{r}\right)^{2} \\
& \Longleftrightarrow s^{2}-2 r s+\frac{m_{B}^{2} r^{2}}{L_{B}^{2}}>0 \\
& \Longleftrightarrow\left(s-\gamma_{1}\right)\left(s-\gamma_{2}\right)>0
\end{aligned}
$$

with $\gamma_{1}:=r(1+\sqrt{1-\delta})>r(1-\sqrt{1-\delta})=: \gamma_{2}>\beta$. Combining the above equivalences with inclusion $s \in(0, \beta]$, we deduce that $\kappa^{2}(1-\delta)<1$, which is the desired inequality. Therefore, the mapping $\Lambda$ is a contraction on the nonempty closed subset $C$ of the Hilbert space $X$ with constant $\kappa(1-\delta)^{\frac{1}{2}}$. From the arbitrariness of $s \in(\rho \alpha, \beta]$, it is easy to see that $\Lambda$ is a contraction on $C$ with constant $(1-\rho \alpha / r)^{-1}(1-\delta)^{\frac{1}{2}}$.

(c) The classical Banach fixed point theorem then guarantees the existence of unique $z_{\eta} \in C$ such that $\Lambda z_{\eta}=z_{\eta}$. Now, putting together this equality and the inclusion (10), we get

$$
-\rho B\left(z_{\eta}-\eta\right) \in N\left(C ; z_{\eta}\right)
$$

or, equivalently (keeping in mind that $N\left(C ; z_{\eta}\right)$ is a cone in $\left.X\right)$,

$$
-B\left(z_{\eta}-\eta\right) \in N\left(C ; z_{\eta}\right) .
$$

It remains to observe that (23) and assumption $\rho \in(0,1)$ (coming from inequality $m_{B}<L_{B}^{2}$ ) imply that

$$
-B\left(z_{\eta}-\eta\right)=\left(z_{\eta}-B(z-\eta)\right)-z_{\eta} \in N\left(C ; z_{\eta}\right) \cap r \mathbb{U}_{X}
$$

This inclusion and Lemma 2.5 entail that

$$
z_{\eta}=\operatorname{proj}_{C}\left(z_{\eta}-B(z-\eta)\right) \text {, }
$$

which concludes the proof. 
We now use Lemma 4.1 to deduce the following result.

Lemma 4.2 Assume $(\mathcal{C})$ and let $B: X \rightarrow X$ be an $m_{B}$-strongly monotone and $L_{B}$-Lipschitz continuous operator with $m_{B}<\min \left\{L_{B}, L_{B}^{2}\right\}$. Moreover, assume that

$$
\alpha:=\sup _{x \in \operatorname{Im}(C)-K}\|B(x)\|_{X}<\rho\left(1-\sqrt{1-\rho m_{B}}\right) r=: \beta \quad \text { with } \rho:=\frac{m_{B}}{L_{B}^{2}} .
$$

Then, for any $\eta \in C(I ; K)$, there exists a unique continuous function $z_{\eta}: I \rightarrow X$ such that

$$
z_{\eta}(t)=\operatorname{proj}_{C(t)}\left(z_{\eta}(t)-B\left(z_{\eta}(t)-\eta(t)\right)\right) \quad \text { for all } t \in I
$$

Proof Let $\eta \in C(I ; K)$. Thanks to Lemma 4.1, we know that for every $t \in I$ there exists a unique element $z_{\eta}(t) \in C(t)$ such that

$$
z_{\eta}(t)=\operatorname{proj}_{C(t)}\left(z_{\eta}(t)-B\left(z_{\eta}(t)-\eta(t)\right)\right)=\operatorname{proj}_{C(t)}\left(z_{\eta}(t)-\rho B\left(z_{\eta}(t)-\eta(t)\right)\right) .
$$

This justifies the claimed existence and uniqueness property. It remains to establish that $z_{\eta}(\cdot)$ is a continuous function. Fix $t \in I$ and consider a sequence $\left(t_{n}\right)_{n \in \mathbb{N}}$ of elements of $I$ which converges to $t$. Due to the closedness of $I$, we obviously have $t \in I$. For each $n \in \mathbb{N}$, denote $C_{n}:=C\left(t_{n}\right), \eta_{n}:=\eta\left(t_{n}\right), \zeta_{n}:=z_{\eta}\left(t_{n}\right)$, and $\omega_{n}:=\zeta_{n}-\rho B\left(\zeta_{n}-\eta_{n}\right)$. Set also $C_{\infty}:=C(t)$, $\eta_{\infty}:=\eta(t), \zeta_{\infty}:=z_{\eta}(t)$, and $\omega_{\infty}:=\zeta_{\infty}-\rho B\left(\zeta_{\infty}-\eta_{\infty}\right)$. With the above notation at hand, it is clear that for every integer $n \in \mathbb{N}$ we have

$$
\zeta_{\infty}=\operatorname{proj}_{C_{\infty}}\left(\omega_{\infty}\right) \text { and } \zeta_{n}=\operatorname{proj}_{C_{n}}\left(\omega_{n}\right)
$$

hence,

$$
\left\|\zeta_{\infty}-\zeta_{n}\right\|_{X} \leq\left\|\operatorname{proj}_{C_{\infty}}\left(\omega_{\infty}\right)-\operatorname{proj}_{C_{n}}\left(\omega_{\infty}\right)\right\|_{X}+\left\|\operatorname{proj}_{C_{n}}\left(\omega_{\infty}\right)-\operatorname{proj}_{C_{n}}\left(\omega_{n}\right)\right\|_{X} .
$$

We now estimate each of the two terms in the right-hand side of (27). We start by setting

$$
u_{\infty}:=\zeta_{\infty}-\eta_{\infty} \quad \text { and } \quad u_{n}:=\zeta_{n}-\eta_{n} \quad \text { for all } n \in \mathbb{N}
$$

It is readily seen that

$$
\left\|u_{\infty}-u_{n}\right\|_{X} \leq\left\|\zeta_{\infty}-\zeta_{n}\right\|_{X}+\left\|\eta_{\infty}-\eta_{n}\right\|_{X} \quad \text { for all } n \in \mathbb{N}
$$

Set $\delta:=\rho m_{B}$. Fix any real $\varepsilon>0$ with $\rho \alpha+\varepsilon<\beta$ and let $s \in(\rho \alpha+\varepsilon, \beta)$. Since $\zeta_{\infty} \in C_{\infty} \subset$ $U_{r}\left(C_{\infty}\right)$, we can use assumption $(\mathcal{C})$ to see that $d\left(\zeta_{\infty}, C_{n}\right) \rightarrow d\left(\zeta_{\infty}, C_{\infty}\right)=0$. Thus, we can find some integer $N \geq 1$ such that

$$
d\left(\zeta_{\infty}, C_{n}\right) \leq \varepsilon \quad \text { for all } n \geq N
$$

Fix for a moment an integer $n \geq N$. We easily observe that

$$
d_{C_{n}}(\omega) \leq d_{C_{n}}\left(\zeta_{\infty}\right)+\rho\left\|B\left(\zeta_{\infty}-\eta_{\infty}\right)\right\|_{X} \leq \varepsilon+\rho \alpha<s
$$


On the other hand, Lemma 4.1(a) guarantees that

$$
d_{C_{n}}\left(\omega_{n}\right) \leq \rho \alpha<s
$$

and the $r$-prox-regularity of $C_{n}$ implies that

$$
\left\|\operatorname{proj}_{C_{n}}\left(\omega_{\infty}\right)-\operatorname{proj}_{C_{n}}\left(\omega_{n}\right)\right\|_{X} \leq \kappa\left\|\omega_{\infty}-\omega_{n}\right\|_{X}
$$

with $\kappa:=(1-s / r)^{-1}$. Using this inequality, the definition of $\omega_{\infty}, \omega_{n}$, and (28), we see that

$$
\begin{aligned}
\left\|\operatorname{proj}_{C_{n}}\left(\omega_{\infty}\right)-\operatorname{proj}_{C_{n}}\left(\omega_{n}\right)\right\|_{X} & \leq \kappa\left\|\zeta_{\infty}-\rho B\left(\zeta_{\infty}-\eta_{\infty}\right)-\zeta_{n}+\rho B\left(\zeta_{n}-\eta_{n}\right)\right\|_{X} \\
& =\kappa\left\|u_{\infty}-u_{n}+\rho\left(B u_{n}-B u_{\infty}\right)+\eta_{\infty}-\eta_{n}\right\|_{X} \\
& \leq \kappa\left\|u_{\infty}-u_{n}+\rho\left(B u_{n}-B u_{\infty}\right)\right\|_{X}+\left\|\eta_{\infty}-\eta_{n}\right\|_{X}
\end{aligned}
$$

Moreover, using the $m_{B}$-strong monotonicity of $B$ and its $L_{B}$-Lipschitz property yields

$$
\begin{aligned}
& \left\|\left(u_{\infty}-u_{n}\right)-\rho\left(B u_{\infty}-B u_{n}\right)\right\|_{X}^{2} \\
& \quad \leq\left\|u_{\infty}-u_{n}\right\|_{X}^{2}-2 \rho\left(u_{\infty}-u_{n}, B u_{\infty}-B u_{n}\right)_{X}+\rho^{2}\left\|B u_{\infty}-B u_{n}\right\|_{X}^{2} \\
& \quad \leq\left(1+\rho^{2} L_{B}^{2}-2 \rho m_{B}\right)\left\|u_{\infty}-u_{n}\right\|_{X}^{2}=(1-\delta)\left\|u_{\infty}-u_{n}\right\|_{X}^{2},
\end{aligned}
$$

or, equivalently,

$$
\left\|u_{\infty}-u_{n}-\rho\left(B u_{\infty}-B u_{n}\right)\right\|_{X} \leq \sqrt{(1-\delta)}\left\|u_{\infty}-u_{n}\right\|_{X} .
$$

Finally, letting $L:=\kappa \sqrt{1-\delta}$ and taking into account inequalities (31), (30), and (29) it follows that

$$
\left\|\operatorname{proj}_{C_{n}}\left(\omega_{\infty}\right)-\operatorname{proj}_{C_{n}}\left(\omega_{n}\right)\right\|_{X} \leq L\left\|\zeta_{\infty}-\zeta_{n}\right\|_{X}+(1+L)\left\|\eta_{\infty}-\eta_{n}\right\|_{X} .
$$

Noting that $L \in(0,1)$ (see Lemma 4.1(b)) and coming back to inequality (27), we see that

$$
(1-L)\left\|\zeta_{n}-\zeta_{\infty}\right\|_{X} \leq\left\|\operatorname{proj}_{C_{\infty}}\left(\omega_{\infty}\right)-\operatorname{proj}_{C_{n}}\left(\omega_{\infty}\right)\right\|_{X}+(1+L)\left\|\eta_{n}-\eta_{\infty}\right\|_{X} .
$$

Next, using inequality

$$
d_{D}\left(\omega_{\infty}\right) \leq d_{D}\left(\zeta_{\infty}\right)+\rho\left\|B\left(\zeta_{\infty}-\theta\right)\right\|_{X}=\rho\left\|B\left(\zeta_{\infty}-\theta\right)\right\|_{X} \leq \rho \alpha<r
$$

it follows that $\omega_{\infty} \in U_{r}\left(C_{\infty}\right)$. Therefore, using assumption $(\mathcal{C})$ and Theorem 2.6, we obtain that

$$
\left\|\operatorname{proj}_{C_{\infty}}\left(\omega_{\infty}\right)-\operatorname{proj}_{C_{n}}\left(\omega_{\infty}\right)\right\|_{X} \rightarrow 0
$$

It remains to use (32) and the continuity of the function $\eta: I \rightarrow K$ to see that $\zeta_{n}=z\left(t_{n}\right) \rightarrow$ $z(t)=\zeta_{\infty}$ in $X$, as $n \rightarrow \infty$. This shows that the function $z_{\eta}: I \rightarrow X$ is continuous and concludes the proof.

The next step is the following. 
Lemma 4.3 Assume (C), ( $\mathcal{A}),(16),(17)$, and (18). Then, for any $\eta \in C(I ; K)$, there exists a unique continuous function $u_{\eta}: I \rightarrow X$ such that

$$
-u_{\eta}(t) \in N\left(C(t) ; A u_{\eta}(t)+\eta(t)\right) \cap s \mathbb{B}_{X} \quad \text { for all } t \in I .
$$

Proof Using Proposition 2.1, it follows that the operator $A^{-1}$ is $m_{A^{-1}}:=\frac{m_{A}}{L_{A}^{2}}$-strongly monotone and $L_{A^{-1}}:=\frac{1}{m_{A}}$-Lipschitz continuous. Note that $m_{A^{-1}}<\min \left\{L_{A^{-1}}, L_{A^{-1}}^{2}\right\}$ and, moreover,

$$
s<\rho\left(1-\sqrt{1-\rho m_{A^{-1}}}\right) r \quad \text { with } \rho:=\frac{m_{A^{-1}}}{L_{A^{-1}}^{2}} .
$$

Let $\eta \in C(I ; K)$ and denote by $z_{\eta} \in C(I ; X)$ the function obtained in Lemma 4.2 with $B:=$ $A^{-1}$. Then

$$
z_{\eta}(t)=\operatorname{proj}_{C(t)}\left(z_{\eta}(t)-u_{\eta}(t)\right) \quad \text { for all } t \in I
$$

with $u_{\eta} \in C(I ; X)$ defined by

$$
u_{\eta}(t):=A^{-1}\left(z_{\eta}(t)-\eta(t)\right) \in s \mathbb{B}_{X} \quad \text { for all } t \in I .
$$

It follows from the definition of proximal normal cone that

$$
\left(z_{\eta}(t)-u_{\eta}(t)\right)-z_{\eta}(t)=-u_{\eta}(t) \in N\left(C(t) ; z_{\eta}(t)\right) \quad \text { for all } t \in I,
$$

and this concludes the proof of the existence part of the lemma.

Now, let $u_{1}, u_{2}: I \rightarrow X$ be two functions such that

$$
-u_{1}(t) \in N\left(C(t) ; A u_{1}(t)+\eta(t)\right) \cap s \mathbb{B}_{X} \quad \text { and } \quad-u_{2}(t) \in N\left(C(t) ; A u_{2}(t)+\eta(t)\right) \cap s \mathbb{B}_{X}
$$

for every $t \in I$. Fix any $t \in I$. Then, for each $i \in\{1,2\}$, we have

$$
A u_{i}(t)+\eta(t) \in C(t)
$$

along with

$$
\left(u_{i}(t), A u_{i}(t)+\eta(t)-v\right)_{X} \leq \frac{\left\|u_{i}(t)\right\|_{X}}{2 r}\left\|A u_{i}(t)+\eta(t)-v\right\|_{X}^{2} \quad \text { for all } v \in C(t) .
$$

This implies that

$$
\begin{aligned}
& \left(u_{1}(t), A u_{1}(t)+\eta(t)-\left(A u_{2}(t)+\eta(t)\right)\right)_{X} \leq \frac{s}{2 r}\left\|A u_{1}(t)-A u_{2}(t)\right\|_{X}^{2}, \\
& \left(u_{2}(t), A u_{2}(t)+\eta(t)-\left(A u_{1}(t)+\eta(t)\right)\right)_{X} \leq \frac{s}{2 r}\left\|A u_{1}(t)-A u_{2}(t)\right\|_{X}^{2},
\end{aligned}
$$

and adding these inequalities yields

$$
\left(u_{1}(t)-u_{2}(t), A u_{1}(t)-A u_{2}(t)\right)_{X} \leq \frac{s}{r}\left\|A u_{1}(t)-A u_{2}(t)\right\|_{X}^{2} .
$$


Using now the $m_{A}$-strong monotonicity and the $L_{A}$-Lipschitz property of the operator $A$, we get

$$
m_{A}\left\|u_{1}(t)-u_{2}(t)\right\|_{X}^{2} \leq \frac{s L_{A}^{2}}{r}\left\|u_{1}(t)-u_{2}(t)\right\|_{X}^{2}
$$

We now use the the assumption $m_{A}>\frac{s L_{A}^{2}}{r}$ to deduce that $u_{1}(t)=u_{2}(t)$.

Lemma 4.3 allows us to consider the operator $\Lambda: C(I ; K) \rightarrow C(I ; K)$ defined by

$$
\Lambda \eta:=\mathcal{S} u_{\eta} \quad \text { for all } \eta \in C(I ; K) \text {. }
$$

We have the following result.

Lemma 4.4 Assume $(\mathcal{C}),(\mathcal{A}),(\mathcal{H}),(16),(17),(18)$, and (19). Then the operator $\Lambda$ has a unique fixed point $\eta^{*} \in C(I ; K)$.

Proof According to Theorem 2.10, it is enough to prove that the operator $\Lambda: C(I ; K) \rightarrow$ $C(I ; K)$ is an almost history-dependent operator. Let $\eta_{1}, \eta_{2} \in C(I ; K)$. Using Lemma 4.3, we find two continuous functions $u_{1}:=u_{\eta_{1}}: I \rightarrow s \mathbb{B}_{X}$ and $u_{2}:=u_{\eta_{2}}: I \rightarrow s \mathbb{B}_{X}$ such that

$$
-u_{1}(t) \in N\left(C(t) ; A u_{1}(t)+\eta_{1}(t)\right) \quad \text { and } \quad-u_{2}(t) \in N\left(C(t) ; A u_{2}(t)+\eta_{2}(t)\right)
$$

for all $t \in I$. Let $\mathcal{J}$ be a nonempty compact subset of $I$ and let $t \in \mathcal{J}$. Using (33) and assumption $(\mathcal{S})$ yields

$$
\begin{aligned}
\left\|\Lambda \eta_{1}(t)-\Lambda \eta_{2}(t)\right\|_{X} & =\left\|\mathcal{S} u_{1}(t)-\mathcal{S} u_{2}(t)\right\|_{X} \\
& \leq l_{\mathcal{J}}^{\mathcal{S}}\left\|u_{1}(t)-u_{2}(t)\right\|_{X}+L_{\mathcal{J}}^{\mathcal{S}} \int_{0}^{t}\left\|u_{1}(s)-u_{2}(s)\right\|_{X} d s .
\end{aligned}
$$

On the other hand, from (34) we see that

$$
A u_{i}(t)+\eta_{i}(t) \in C(t)
$$

for each $i \in\{1,2\}$ and, therefore,

$$
\left(u_{i}(t), A u_{i}(t)+\eta_{i}(t)-v\right)_{X} \leq \frac{s}{2 r}\left\|A u_{i}(t)+\eta_{i}(t)-v\right\|_{X}^{2} \quad \text { for all } v \in C(t) .
$$

Taking $i=1$ and $v:=A u_{2}(t)+\eta_{2}(t)$ in the above estimate yields

$$
\left(u_{1}(t), A u_{1}(t)+\eta_{1}(t)-A u_{2}(t)-\eta_{2}(t)\right)_{X} \leq \frac{s}{2 r}\left\|A u_{1}(t)+\eta_{1}(t)-A u_{2}(t)-\eta_{2}(t)\right\|_{X}^{2} .
$$

Similarly, taking $i=2$ and $v:=A u_{1}(t)+\eta_{1}(t)$, we get

$$
\left(u_{2}(t), A u_{2}(t)+\eta_{2}(t)-A u_{1}(t)-\eta_{1}(t)\right)_{X} \leq \frac{s}{2 r}\left\|A u_{2}(t)+\eta_{2}(t)-A u_{1}(t)-\eta_{1}(t)\right\|_{X}^{2} .
$$


Adding the previous two inequalities, we find that

$$
\begin{aligned}
& \left(u_{1}(t)-u_{2}(t), A u_{1}(t)-A u_{2}(t)\right)_{X} \\
& \quad \leq\left(u_{1}(t)-u_{2}(t), \eta_{2}(t)-\eta_{1}(t)\right)_{X}+\frac{s}{r}\left\|A u_{2}(t)+\eta_{2}(t)-A u_{1}(t)-\eta_{1}(t)\right\|_{X}^{2} .
\end{aligned}
$$

Fix $\varepsilon>0$ such that $m_{A}-\frac{\varepsilon^{2}}{2}-\frac{2 s L_{A}^{2}}{r}>0$. Then, using the Cauchy-Schwarz inequality and the elementary inequality $2 a b \leq(\varepsilon a)^{2}+\left(\frac{b}{\varepsilon}\right)^{2}$ valid for every real $a, b$, we obtain that

$$
\left(u_{1}(t)-u_{2}(t), \eta_{2}(t)-\eta_{1}(t)\right)_{X} \leq \frac{\varepsilon^{2}}{2}\left\|u_{1}(t)-u_{2}(t)\right\|_{X}^{2}+\frac{1}{2 \varepsilon^{2}}\left\|\eta_{1}(t)-\eta_{2}(t)\right\|_{X}^{2}
$$

On the other hand, note that

$$
\begin{aligned}
\left\|A u_{1}(t)+\eta_{1}(t)-A u_{2}(t)-\eta_{2}(t)\right\|_{X}^{2}= & \left\|A u_{1}(t)-A u_{2}(t)\right\|_{X}^{2}+\left\|\eta_{1}(t)-\eta_{2}(t)\right\|_{X}^{2} \\
& +2\left(A u_{1}(t)-A u_{2}(t), \eta_{1}(t)-\eta_{2}(t)\right)_{X} \\
\leq & L_{A}^{2}\left\|u_{1}(t)-u_{2}(t)\right\|_{X}^{2}+\left\|\eta_{1}(t)-\eta_{2}(t)\right\|_{X}^{2} \\
& +2 L_{A}\left\|u_{1}(t)-u_{2}(t)\right\|\left\|\eta_{1}(t)-\eta_{2}(t)\right\|_{X} \\
\leq & L_{A}^{2}\left\|u_{1}(t)-u_{2}(t)\right\|_{X}^{2}+\left\|\eta_{1}(t)-\eta_{2}(t)\right\|_{X}^{2} \\
& +L_{A}^{2}\left\|u_{1}(t)-u_{2}(t)\right\|_{X}^{2}+\left\|\eta_{1}(t)-\eta_{2}(t)\right\|_{X}^{2} \\
= & 2 L_{A}^{2}\left\|u_{1}(t)-u_{2}(t)\right\|_{X}^{2}+2\left\|\eta_{1}(t)-\eta_{2}(t)\right\|_{X}^{2} .
\end{aligned}
$$

Therefore, setting $a_{\varepsilon}:=m_{A}-\frac{\varepsilon^{2}}{2}-\frac{2 s L_{A}^{2}}{r}, b_{\varepsilon}:=\frac{1}{2 \varepsilon^{2}}+\frac{2 s}{r}$ and taking into account the strong monotonicity of the operator $A$, we find that

$$
\left\|u_{1}(t)-u_{2}(t)\right\|_{X}^{2} \leq \frac{b_{\varepsilon}}{a_{\varepsilon}}\left\|\eta_{1}(t)-\eta_{2}(t)\right\|_{X}^{2}
$$

Now, choosing $\varepsilon:=\sqrt{m_{A}}$, we get

$$
\left\|u_{1}(t)-u_{2}(t)\right\|_{X} \leq c\left\|\eta_{1}(t)-\eta_{2}(t)\right\|_{X}
$$

with $c:=\left(\frac{1}{2 m_{A}}+\frac{2 s}{r}\right)^{1 / 2}\left(\frac{m_{A}}{2}-\frac{2 s L_{A}^{2}}{r}\right)^{-1 / 2}$. Substituting this inequality in (35) yields

$$
\begin{aligned}
\left\|\Lambda \eta_{1}(t)-\Lambda \eta_{2}(t)\right\|_{X} & =\left\|\mathcal{S} u_{1}(t)-\mathcal{S} u_{2}(t)\right\|_{X} \\
& \leq c l_{\mathcal{J}}^{\mathcal{S}}\left\|\eta_{1}(t)-\eta_{2}(t)\right\|_{X}+c L_{\mathcal{J}}^{\mathcal{S}} \int_{0}^{t}\left\|\eta_{1}(s)-\eta_{2}(s)\right\|_{X} d s .
\end{aligned}
$$

We now invoke the smallness assumption (19) to obtain that the operator $\Lambda$ is an almost history-dependent operator. It remains to apply Theorem 2.10 to complete the proof.

We are now in a position to provide the proof of Theorem 3.1. 
Proof Let $\eta^{*} \in C(I ; K)$ be the fixed point of the operator $\Lambda$, and let $u^{\star}:=u_{\eta^{\star}} \in C(I ; X)$ be the function given by Lemma 4.3 with $\eta:=\eta^{\star}$. So, we have

$$
-u^{\star}(t) \in N(C(t) ; A u(t)+\eta(t)) \cap s \mathbb{B}_{X} \quad \text { for all } t \in I .
$$

This inclusion combined with equality $\eta^{\star}=\Lambda \eta^{\star}=\mathcal{S} u^{\star}$ implies that

$$
-u^{\star}(t) \in N\left(C(t) ; A u^{\star}(t)+\mathcal{S} u^{\star}(t)\right) \cap s \mathbb{B}_{X} \quad \text { for all } t \in I,
$$

which shows that $u^{\star}$ is a solution to Problem 2. This proves the existence part of Theorem 3.1. The uniqueness part is a direct consequence of the uniqueness of the fixed point of the operator $\Lambda$.

\section{An example}

In this section we provide an example of Problem 2 for which our abstract results work. To this end we consider two elements $\boldsymbol{a}_{1}, \boldsymbol{a}_{2} \in \mathbb{S}^{d}$ and three real constants $g_{1}, g_{2}$, and $k$ such that

$$
\begin{aligned}
& g_{1}, g_{2}, k>0, \\
& k>g_{2}, \\
& \left\|a_{1}-a_{2}\right\|>g_{1}+k, \\
& \frac{\left\|\boldsymbol{a}_{1}\right\|+g_{1}}{\left\|\boldsymbol{a}_{1}-\boldsymbol{a}_{2}\right\|-g_{1}-g_{2}}<\frac{\sqrt{7}}{32} .
\end{aligned}
$$

We now introduce the sets

$$
\begin{aligned}
& C_{1}=\left\{\boldsymbol{x} \in \mathbb{S}^{d}:\left\|\boldsymbol{x}-\boldsymbol{a}_{1}\right\| \leq g_{1}\right\}, \\
& C_{2}=\left\{\boldsymbol{x} \in \mathbb{S}^{d}:\left\|\boldsymbol{x}-\boldsymbol{a}_{2}\right\| \leq g_{2}\right\}, \\
& C(t)=C_{1} \cup C_{2} \quad \forall t \in I, \\
& K=\left\{\boldsymbol{x} \in \mathbb{S}^{d}:\left\|\boldsymbol{x}-\boldsymbol{a}_{2}\right\| \leq k\right\} .
\end{aligned}
$$

For the sake of simplicity, we only consider the setting where $C(t)$ is autonomous, i.e., independent of time $t$. Nevertheless, we mention that the result below in this section can be easily extended to the case when $g_{1}, g_{2}$ are real-valued positive functions depending on $t \in I$ and, in this case, the set $C(t)$ will depend on $t$. We denote by $P_{K}: \mathbb{S}^{d} \rightarrow K$ the projection operator on the closed convex set $K$ and consider the following inclusion problem.

Problem 4 Find a continuous function $\boldsymbol{u}: I \rightarrow X$ such that

$$
-\boldsymbol{u}(t) \in N\left(C(t) ; \boldsymbol{u}(t)+P_{K}\left(\int_{0}^{t} \boldsymbol{u}(s) d s\right)\right) \quad \text { for all } t \in I
$$

We have the following existence and uniqueness result. 
Theorem 5.1 Assume (36)-(39). Then Problem 4 has at least a solution $\boldsymbol{u}(\cdot)$. Moreover, the solution takes values in $s \mathbb{B}_{\mathbb{S}^{d}}:=\left\{s \boldsymbol{b}: \boldsymbol{b} \in \mathbb{B}_{\mathbb{S}^{d}}\right\}$ with $s:=\left\|\boldsymbol{a}_{1}\right\|+g_{1}$ and is the unique solution of Problem 4 with this property.

Proof We apply Corollary 3.3 on the space $X=\mathbb{S}^{d}$ with $C(t)$ defined by (40)-(42), $K$ defined by (43), and the operators $A, \mathcal{S}$ given by

$$
\begin{aligned}
& A \boldsymbol{u}=\boldsymbol{u} \quad \forall \boldsymbol{u} \in \mathbb{S}^{d} \\
& \mathcal{S} \boldsymbol{u}(t)=P_{K}\left(\int_{0}^{t} \boldsymbol{u}(s) d s\right) \quad \forall \boldsymbol{u} \in C\left(I ; \mathbb{S}^{d}\right) .
\end{aligned}
$$

First, we remark that the sets $C_{1}$ and $C_{2}$ are convex and, therefore they are $\infty$-prox regular. Moreover, using assumptions (37), (38), we see that

$$
g:=\inf _{\left(\boldsymbol{c}_{1}, \boldsymbol{c}_{2}\right) \in C_{1} \times C_{2}}\left\|\boldsymbol{c}_{1}-\boldsymbol{c}_{2}\right\|>0=\left\|\boldsymbol{a}_{1}-\boldsymbol{a}_{2}\right\|-g_{1}-g_{2}>0 .
$$

Therefore, Lemma 2.7 guarantees that for each $t \in I$ the set $C(t)$ is $r$-prox-regular with

$$
r=\frac{1}{2}\left(\left\|\boldsymbol{a}_{1}-\boldsymbol{a}_{2}\right\|-g_{1}-g_{2}\right) .
$$

In addition, since $C(t)$ does not depend on $t$, it follows that convergence (15) holds. We conclude from here that assumption $(\mathcal{C})$ is satisfied.

On the other hand, it is obvious to see that the operator $A$ satisfies condition $(\mathcal{A})$ with $m_{A}=1$ and $L_{A}=1+\varepsilon$ for any $\varepsilon>0$. Moreover, the operator $\mathcal{S}$ defined by (45) is historydependent operator (keeping in mind that $P_{K}$ is 1-Lipschitz on $\mathbb{S}^{d}$ ). In addition, inequality (16) is obviously satisfied.

We now show that with a convenient choice of $\varepsilon$, conditions (17) and (18) are satisfied. To this end we use (37) and (38) to see that $\operatorname{Im}(C)-K=\left(C_{1} \cup C_{2}\right)-K=C_{1}$ and, therefore,

$$
s:=\sup _{\boldsymbol{x} \in \operatorname{Im}(C)-K}\left\|A^{-1} \boldsymbol{x}\right\|=\sup _{\boldsymbol{x} \in C_{1}}\|\boldsymbol{x}\|=\left\|\boldsymbol{a}_{1}\right\|+g_{1} .
$$

Hence, using (46), (47) and equalities $m_{A}=1, L_{A}=1+\varepsilon$, it follows that conditions (17), (18) are equivalent with the inequalities

$$
\begin{aligned}
& \frac{\left\|\boldsymbol{a}_{1}\right\|+g_{1}}{\left\|\boldsymbol{a}_{1}-\boldsymbol{a}_{2}\right\|-g_{1}-g_{2}}<\frac{1}{2(1+\varepsilon)^{2}}\left(1-\sqrt{1-\frac{1}{(1+\varepsilon)^{4}}}\right), \\
& \frac{\left\|\boldsymbol{a}_{1}\right\|+g_{1}}{\left\|\boldsymbol{a}_{1}-\boldsymbol{a}_{2}\right\|-g_{1}-g_{2}}<\frac{1}{8(1+\varepsilon)^{2}},
\end{aligned}
$$

respectively. Next, an elementary calculus reveals that

$$
x>\frac{\sqrt{7}}{4} \quad \Longrightarrow \quad \frac{\sqrt{7}}{32}<\frac{x}{8}<\frac{x}{2}\left(1-\sqrt{1-x^{2}}\right),
$$

and, using this inequality with $x=\frac{1}{(1+\varepsilon)^{2}}$, we deduce that

$$
0<\varepsilon<\frac{2}{\sqrt{\sqrt{7}}}-1 \quad \Longrightarrow \quad \frac{\sqrt{7}}{32}<\frac{1}{8(1+\varepsilon)^{2}}<\frac{1}{2(1+\varepsilon)^{2}}\left(1-\sqrt{1-\frac{1}{(1+\varepsilon)^{4}}}\right) .
$$


Choose now $0<\varepsilon<\frac{2}{\sqrt[4]{7}}-1$. Then, using (39) and (50), we find that (48) and (49) hold, which implies that conditions (17) and (18) are satisfied, too. Theorem 5.1 is now a direct consequence of Corollary 3.3 .

\section{Acknowledgements}

The first author is indebted to Assalé Adjé for helpful discussions on prox-regularity.

\section{Funding}

This research was supported by the European Union's Horizon 2020 Research and Innovation Programme under the Marie Sklodowska-Curie Grant Agreement No. 823731 CONMECH.

\section{Abbreviations}

Not applicable.

Availability of data and materials

Not applicable.

\section{Declarations}

Ethics approval and consent to participate

Not applicable.

Consent for publication

Not applicable.

Competing interests

The authors declare that they have no competing interests.

Authors' contributions

FN and MS contributed equally in writing this article. All authors read and approved the final manuscript.

\section{Authors' information}

Not applicable.

\section{Publisher's Note}

Springer Nature remains neutral with regard to jurisdictional claims in published maps and institutional affiliations.

Received: 5 October 2021 Accepted: 13 January 2022 Published online: 14 February 2022

\section{References}

1. Adly, S., Haddad, T.: An implicit sweeping process approach to quasistatic evolution variational inequalities. SIAM J. Math. Anal. 50, 761-778 (2018)

2. Adly, S., Nacry, F., Thibault, L.: Preservation of prox-regularity of sets with applications to constrained optimization. SIAM J. Optim. 26, 448-473 (2016)

3. Adly, S., Nacry, F., Thibault, L.: Discontinuous sweeping process with prox-regular sets. ESAIM Control Optim. Calc. Var. 23, 1293-1329 (2017)

4. Adly, S., Nacry, F., Thibault, L.: Prox-regularity approach to generalized equations and image projection. ESAIM Control Optim. Calc. Var. 24, 677-708 (2018)

5. Adly, S., Sofonea, M.: Time-dependent inclusions and sweeping processes in contact mechanics. Z. Angew. Math. Phys. 70(2), Paper No. 39, 19 pp. (2019)

6. Cao, T.H., Mordukhovich, B.S.: Optimal control of a nonconvex perturbed sweeping process. J. Differ. Equ. 266 1003-1050 (2019)

7. Castaing, C., Ibrahim, A.G., Yarou, M.: Some contributions to nonconvex sweeping process. J. Nonlinear Convex Anal. $10,1-20(2009)$

8. Chemetov, N., Monteiro Marques, M.D.P.: Non-convex quasi-variational differential inclusions. Set-Valued Anal. 15, 209-221 (2007)

9. Clarke, F.H.: Optimization and Nonsmooth Analysis, 2nd edn. Classics in Applied Mathematics, vol. 5. SIAM, Philadelphia (1990)

10. Colombo, G., Goncharov, V.V.: The sweeping processes without convexity. Set-Valued Anal. 7, 357-374 (1999)

11. Colombo, G., Thibault, L.: Prox-regular sets and applications. In: Handbook of Nonconvex Analysis and Applications, pp. 99-182. Int. Press, Somerville (2010)

12. Edmond, J.F., Thibault, L.: BV solutions of nonconvex sweeping process differential inclusions with perturbation. J. Differ. Equ. 226, 135-179 (2006)

13. Haddad, T.: Nonconvex differential variational inequality and state-dependent sweeping process. J. Optim. Theory Appl. 159, 386-398 (2013)

14. Jourani, A., Vilches, E.: Positively $\alpha$-far sets and existence results for generalized perturbed sweeping processes. J. Convex Anal. 23, 775-821 (2016) 
15. Monteiro Marques, M.D.P.: Differential Inclusions in Nonsmooth Mechanical Problems. Shocks and Dry Friction. Progress in Nonlinear Differential Equations and Their Applications, vol. 9. Birkhäuser, Basel (1993)

16. Mordukhovich, B.S.: Variational Analysis and Applications. Springer Monographs in Mathematics. Springer, Cham (2018)

17. Moreau, J.J: Rafle par un convexe variable I. In: Travaux Sém. Anal. Convexe Montpellier (1971), Exposé 15

18. Moreau, J.J.: Evolution problem associated with a moving convex in a Hilbert space. J. Differ. Equ. 26, 347-374 (1977)

19. Nacry, F., Sofonea, M.: A class of nonlinear inclusions and sweeping processes in solid mechanics. Acta Appl. Math. $171,16(2021)$

20. Nacry, F., Sofonea, M.: A history-dependent sweeping processes in contact mechanics. J. Convex Anal. 29 (2022, to appear)

21. Nacry, F., Thibault, L.: Regularization of sweeping process: old and new. Pure Appl. Funct. Anal. 4, 59-117 (2019)

22. Panagiotopoulos, P.D.: Inequality Problems in Mechanics and Applications. Birkhäuser, Boston (1985)

23. Poliquin, R.A., Rockafellar, R.T., Thibault, L.: Local differentiability of distance functions. Transl. Am. Math. Soc. 352, $5231-5249(2000)$

24. Rockafellar, R.T., Wets, R.J.-B.: Variational Analysis. Springer, Berlin (1997)

25. Sofonea, M., Matei, A.: Mathematical Models in Contact Mechanics. London Mathematical Society Lecture Note Series, vol. 398. Cambridge University Press, Cambridge (2012)

26. Sofonea, M., Migórski, S.: Variational-Hemivariational Inequalities with Applications. Pure and Applied Mathematics. Chapman \& Hall/CRC Press, Boca Raton-London (2018)

27. Thibault, L.: Sweeping process with regular and nonregular sets. J. Differ. Equ. 193, 1-26 (2003)

28. Thibault, L.: Unilateral variational analysis in Banach spaces (to appear)

29. Venel, J.: A numerical scheme for a class of sweeping processes. Numer. Math. 118, 367-400 (2011)

\section{Submit your manuscript to a SpringerOpen ${ }^{\circ}$ journal and benefit from:}

- Convenient online submission

- Rigorous peer review

- Open access: articles freely available online

- High visibility within the field

- Retaining the copyright to your article

Submit your next manuscript at $\gg$ springeropen.com 